\title{
Diagnosis and treatment of internal hydrocephalus in dogs in relation to our own observations
}

\author{
OLIWIER TEODOROWSKI, PIOTR TEODOROWSKI, MICHAŁ KIONA, MARTYNA LOSKOT, \\ STANISŁAW WINIARCZYK*, ALICJA WÓJCIK*, ŁUKASZ ADASZEK*
}

\author{
Veterinary Clinic "Teodorowscy" in Mikołów, Poland \\ *Department of Epizootiology and Clinic of Infectious Diseases, Faculty of Veterinary Medicine, \\ University of Life Sciences in Lublin, 30 Głęboka St, 20-612 Lublin, Poland
}

Teodorowski O., Teodorowski P., Kiona M., Loskot M., Winiarczyk S., Wójcik A., Adaszek Ł. Diagnosis and treatment of internal hydrocephalus in dogs in relation to our own observations

Summary

Hydrocephalus is a congenital or acquired neurological disorder in dogs, associated with the excessive accumulation of cerebrospinal fluid (CSF). The factors contributing to hydrocephalus development are diverse and not always possible to determine. The aim of this article was to present two cases of the diagnosis and treatment of internal hydrocephalus in dogs. The observations included animals with neurological disorders of unknown origin. Internal hydrocephalus was diagnosed based on the results of magnetic resonance imaging, and the treatment of both patients comprised ventricular brain drainage. The surgical treatment was fully effective for one dog. For the other dog, a short-term improvement was followed by deterioration in condition due to occlusion of the ventriculoperitoneal drain, and the owners decided to euthanize the animal. The analysis of our cases led to the conclusion that congenital hydrocephalus is a disorder that challenges veterinarians in terms of diagnosis and therapy, due to limited access to advanced imaging techniques such as MRI or CT, and from the complicated nature of the surgical treatment. However, it should be noted that if the disorder is correctly diagnosed and ventricular brain drainage is adequate, the chances for successful functioning are significant.

Keywords: Internal hydrocephalus, ventricular drainage, MRI, dogs

Hydrocephalus is an excessive intracranial accumulation of cerebrospinal fluid in the ventricular system or subarachnoid space (11). Physiologically, the cerebrospinal fluid (CSF) protects, nourishes and supports the functioning of the central nervous system. It is produced mainly by the choroid plexuses of the brain ventricles and by the ependymal cells that form the lining of the ventricular system. CSF production is a process requiring energy consumption and takes place at a rate of about $0.03-0.5 \mathrm{~mL} / \mathrm{min}$. The CSF circulates in the ventricular system of the brain, passing through the lateral ventricles, the third ventricle and the cerebral aqueduct to the fourth ventricle, from where it passes through the lateral apertures into the subarachnoid space of the brain and spinal cord. Resorption of the cerebrospinal fluid takes place through the arachnoid villi in a passive process that does not require energy expenditure. A disturbance in the circulation or balance between the production and resorption of CSF can lead to the excessive accumulation of fluid and consequently to the development of hydrocephalus. This disorder is classified in various ways, depending on the anatomical position (internal or external hydrocephalus), aetiological factors or origin (acquired, congenital, communicat- ing, non-communicating, or idiopathic) or the pressure difference (normo- and hypertensive) (4).

There are various causes of hydrocephalus in dogs. As a rule, they are associated with pathological stenoses within the ventricular system of the brain resulting from malformations or inflammatory changes in the prenatal period. The breeds that are predisposed to the development of congenital hydrocephalus are: Bulldog, Yorkshire Terrier, Chihuahua, Maltese, Pomeranian, Pekingese and Boston Terrier (8).

Acquired internal hydrocephalus can be of two types: compensatory hydrocephalus, related to the loss of brain tissue and subsequent filling of the free space with cerebrospinal fluid (e.g. post-traumatic associated with necrosis or post-infarction volume reduction associated with scarring), and hydrocephalus, caused by obstruction in the spaces where the CSF circulates (4).

Taking into account the site of the blockage, hydrocephalus caused by obstruction can be subdivided into communicating or non-communicating. Obstruction within the ventricular system or within the lateral apertures causes excessive accumulation of CSF in the system; such hydrocephalus is called "non-communicating" because there is no fluid flow 
between the ventricular system and the subarachnoid space. Hydrocephalus is called "communicating" when fluid flow in the subarachnoid space is blocked or its absorption by the arachnoid villi is reduced, but the flow between the cerebral ventricular system and the subarachnoid space is preserved $(4,12)$.

Dogs with hydrocephalus are rare in veterinary clinics, and the diagnosis and treatment of this disorder is a challenge for veterinary surgeons. For this reason, the purpose of this article is to present case studies that include the diagnosis and treatment of internal hydrocephalus in dogs based on our own observations.

\section{Material and methods}

Animals used in the study. The study included two dogs with internal hydrocephalus. The first $(\operatorname{dog} 1)$ was an eighteen-month male Chihuahua referred to the clinic with sudden balance disturbances. The dog had seizures, movement disorders and loss of consciousness. Despite pharmacological treatment, including fluid therapy, steroid therapy (prednisone: Encorton, $2.5 \mathrm{mg} / \mathrm{kg}$ ), phenobarbital therapy (Luminalum, $10 \mathrm{mg} / \mathrm{kg}$ ), its condition did not improve and therefore it was referred for further neurological diagnostics. On admission to the clinic, the dog showed signs of disorientation and disturbances of consciousness, frequent epileptic seizures, circus movements, and aimless pressure forward. A neurological examination showed reduced cranial nerve reflexes, pupil dilation with preserved reaction to light, and normal swallowing reflex, although the dog was unable to drink and eat without assistance. The dog's skull was enlarged and distorted. The blood count and blood serum chemistry showed no abnormalities. Parasitological examination of the faeces excluded the presence of parasites. The dog had received vaccination against infectious diseases and had been subjected to regular prophylaxis against ectoparasites as well as internal parasites. The dog had not suffered any trauma. Because the cause of the disturbances observed in the dog was difficult to determine, it was referred for magnetic resonance imaging.

The second $\operatorname{dog}(\operatorname{dog} 2)$ included in the observation was an eight-year-old sterilised female Yorkshire Terrier, undergoing magnetic resonance imaging due to increasing neurological symptoms. Since the moment of adoption (2 years previously), it had demonstrated impaired movement: it ran into obstacles, made circus movements to the right and showed signs of confusion. No abnormalities were found in blood tests. The treatment including antibiotic therapy (amoxicillin/clavulanic acid: Synulox, $8.75 \mathrm{mg} / \mathrm{kg}$ ), steroid therapy (prednisone: Encorton $2.5 \mathrm{mg} / \mathrm{kg}$ ) and drugs improving nerve conduction (galantamini hydrobromidum: Nivalin, $1 \mathrm{mg} / \mathrm{kg}$ ), which were not effective.

On admission to the clinic, the dog was conscious but had impaired movement and balance disturbances. After gently touching the dog on its side, it fell over. We also noted a slightly reduced menace reflex on the left side and decreased reaction of the right pupil.

Magnetic resonance imaging. The MRI of the head in both dogs was performed with ESAOTE MR-Grande (0.2 Tesla field) using coil 2 for Spin Echo T1-weighted images, FSE T2-weighted images and FAST FLAIR, in the axial, frontal and sagittal planes, without contrast enhancement.
The dogs were premedicated with butorphanol (Torbugesic, Zoetis, $0.1 \mathrm{mg} / \mathrm{kg}$ ) and dexmedetomidine (Dexdomitor, Orion, $17.5 \mu \mathrm{g} / \mathrm{kg}$ ) administered simultaneously by injection. They were placed in the sternal position for the examination.

Ventricular drainage. The dog was placed in a sternal position. In the back of the skull, a hole was made at an angle of $15^{\circ}$. Then the ventricular drain was inserted slowly until the first drop of CSF appeared. Subsequently, the drain was inserted under the skin in the dorsal part of the neck, reaching the level of the last ribs, and placed in the abdominal cavity. In the final stage, a valve of the correct size was inserted to prevent the penetration of microorganisms into the brain.

\section{Results and discussion}

Magnetic resonance imaging results. In $\operatorname{dog} 1$, the magnetic resonance imaging of the head showed a very significant enlargement of the lateral, third and fourth ventricles, and the connecting structures. The lateral ventricles in the widest parts on the cross-section perpendicular to the base of the skull measured $49 \times 55 \mathrm{~mm}$. In addition, a very strong compression of the remaining parts of the brain by the dilated ventricles was observed. The cerebrum was strongly pushed down, with the tissue thickness ranging from 1 to $5 \mathrm{~mm}$. There was a strong pressure on the brain stem, and the cerebellum was deformed, pushed down towards the foramen magnum. The examination did not allow us to determine the root cause of the diagnosed internal hydrocephalus.

In $\operatorname{dog} 2$, the MRI of the head demonstrated a significant degree of hydrocephalus with enlargement of all ventricles, with the abnormalities more pronounced on the right hand side (Fig. 1). A thinning and atrophy

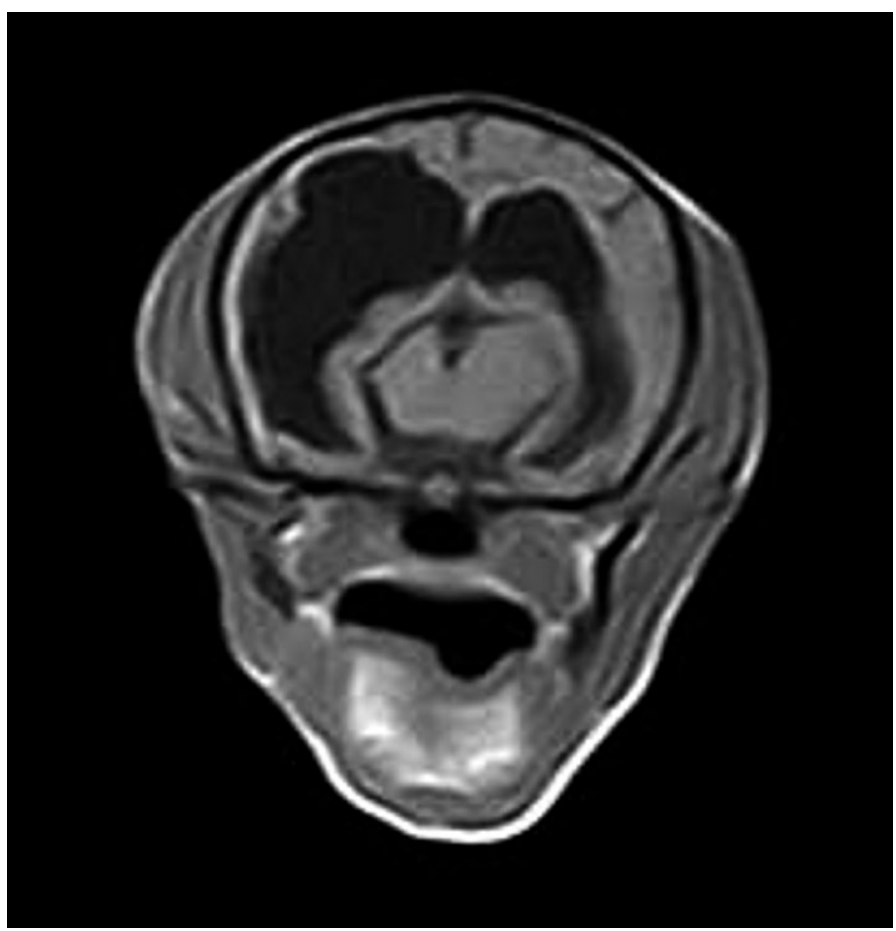

Fig. 1. MRI of the head of $\operatorname{dog} 2$ demonstrated a significant degree of hydrocephalus with enlargement of all ventricles, with the abnormalities more pronounced on the right side 
of the cortical part of the brain was observed, with an increase in the signal characteristic of inflammation and a change in the signal characteristic of the periventricular extravasation. Asymmetry of the thalamus was also visible; however, the cause of the hydrocephalus was not found, even after the contrast examination.

Because of the very advanced disorder, dog 1 received inpatient treatment and was pharmacologically prepared for ventricular drainage. After the implementing diuretic therapy with mannitol (Mannitol $20 \%$, at a dose of $2 \mathrm{~g} / \mathrm{kg}$ a day, within 15-20 minutes), a rapid improvement in the dog's condition was observed. On the second day of the therapy the seizures ceased, and the dog was able to move independently, although movement was still uncoordinated and hypermetry was observed. It was able to eat and drink water by itself. After five days of diuretic therapy, its condition improved significantly, and the decision concerning drainage was made. A set dedicated for paediatric neurosurgery (Medtronic Minneapolis USA) was used; it consisted of a ventricular catheter with a mandrin, a peritoneal drain and a lowpressure CSF Ultra Small Valve. After the procedure, the condition of the dog gradually improved, although there were still abnormalities in its movement and coordination. There was a slight hypermetric response in four limbs, the gait was shaky, but no seizures or forced movements were reported. Five days after the surgery, magnetic resonance imaging was performed. There was a slight decrease in the amount of cerebrospinal fluid; however, the deformations of the previously described parts of the brain, especially in the cerebellum, were largely maintained. The dog was discharged from hospital due to the combination of its well-being and its ability to function independently. About a month after leaving the hospital, the dog's condition was satisfactory. The neurological deficits described earlier were maintained but did not significantly affect its quality of life. It was able to eat and drink by itself, it was energetic and prone to physical activity. About 2 months after the surgery, the dog's condition deteriorated. There were recurrent epileptic seizures, motor dysfunction, consciousness disorders and forced movements. There was a strong decrease in body temperature to $33.4^{\circ} \mathrm{C}$. It was re-hospitalized and received anticonvulsant treatment with diazepam (Relanium, Polfa Warszawa S.A., $0.3 \mathrm{mg} / \mathrm{kg}$ ), steroid therapy with dexamethasone (Rapidexon, Eurovet Animal Health BV, $2 \mathrm{mg} / \mathrm{kg}$ ) and fluid therapy. After the first day of treatment, the condition improved: the epileptic seizures subsided, the intensity of forced movements decreased, and the level of awareness increased. It was still unable to move on its own or take food and water; however, the swallowing reflex was preserved. The blood count and blood serum chemistry showed no abnormalities. Steroid therapy and symptomatic treatment was continued for 10 consecutive days, with no significant effects. The owner did not agree to perform a control MR imaging and decided to euthanize the dog due to its poor condition and lack of effects from the pharmacological treatment.
Because dog 2 was diagnosed with internal hydrocephalus using MR imaging, two days after the examination it received fluid therapy with isotonic crystalloids, steroid therapy with dexamethasone (Rapidexon, $2 \mathrm{mg} / \mathrm{kg}$ ), loop diuretics (Furosemidum, $2 \mathrm{mg} / \mathrm{kg}$ ) and underwent a ventricular drain implantation procedure to decompress the brain ventricles. A set dedicated for paediatric neurosurgery (Medtronic Minneapolis USA) was used; it consisted of a ventricular catheter with a mandrin, a peritoneal drain and a low-pressure CSF Ultra Small Valve. After the procedure, a gradual improvement in the dog's condition was observed; it began to move better and became more active. During the follow up visits, it easily followed its guardian. Due to the ongoing clinical improvement, the owners decided against a control MRI. The good condition of the dog has been maintained for 3 years.

The pathological influence mechanism of the emerging internal hydrocephalus on the brain can be divided into primary and secondary change stages. Primary changes developing in the initial phase of the hydrocephalus are primarily associated with enlargement of the cerebral ventricular system and the pressure exerted on the surrounding structures. With progression of the disease, ischaemia and oedema of the interstitial periventricular tissues and the grey matter of the cerebral cortex develop. A loss in the lining of the ventricular system, the formation of fissures and diverticula and disorders of the blood-brain barrier are also observed. Secondary mechanisms that accompany internal hydrocephalus are: tissue necrosis, gliosis, and metabolic and conductivity disorders within the white matter (9). All these disorders, both secondary and primary, manifested in the form of clinical symptoms that were observable in both dogs.

Neurological deficiencies may appear, depending on the case, both as symptoms of excessive stimulation of the central nervous system and deep depression (13). Motor disorders and convulsions were observed in the dogs, although visual and hearing disorders, forced and circus movements, disturbances of consciousness and balance disorders may also develop in the internal hydrocephalus. In addition, individuals with congenital hydrocephalus have distorted, bloated skulls (similar to that of dog 1) and are usually characterized by a finer body structure compared to other individuals of the same age $(3,14)$. Because of the nonspecific and wide spectrum of observed neurological symptoms, it is necessary to use additional advanced diagnostic methods to establish the final diagnosis of hydrocephalus.

The first diagnostic method is cranial ultrasound. It is performed in young dogs with a non-ossified fontanel. The advantage of this examination is the ability to perform it without the need for general anaesthesia, while the obvious disadvantage is that it cannot be performed in all individuals with suspected hydrocephalus $(1,13)$.

Computed tomography and magnetic resonance imaging give a better visualization of hydrocephalus on the basis of desired cross-sections, and usually provide 
appropriate measurements of the ventricles of the brain, which makes it possible to differentiate hydrocephalus from a dilated ventricular system of the brain (6).

Magnetic resonance imaging, especially after the administration of contrast, can demonstrate the causes of hydrocephalus limiting cerebrospinal fluid outflow from the ventricular system. Abnormalities such as cysts, granulomas, abscesses or tumours are easier to identify due to a higher image resolution in relation to computed tomography. A disadvantage of magnetic resonance imaging is the need to use a long-lasting general anaesthesia $(4,13)$.

The longer duration of the MRI examination, and thus longer anaesthesia, in comparison to computed tomography, especially in cases involving dogs with suspected hydrocephalus and with co-existing cardiorespiratory insufficiency, indicate the favourable choice of the latter diagnostic method.

The methods of treatment for internal hydrocephalus can be divided into pharmacological and surgical. They are often combined by preparing patients pharmacologically, to improve their general condition, before proceeding with the surgical procedure.

Pharmacotherapy is based primarily on the symptomatic treatment of the neurological symptoms (e.g. anticonvulsant therapy) and on the attempt to reduce intracranial pressure by limiting the production of cerebrospinal fluid. For this purpose, furosemide, acetazolamide (carbonic anhydrase inhibitor) and glucocorticoids, e.g. prednisone (13), are most commonly used. Pharmacological therapy usually brings temporary improvement and carries a risk of side effects, especially with long-term use, in the form of a waterelectrolyte imbalance. A case has been reported where acetazolamide therapy did not result in any clinical improvement (10).

Potentially the best long-lasting healing effect is obtained from surgical treatment using a ventricular shunt. In this method, a drain provided with a special valve removes excess cerebrospinal fluid, directing it to a different space in the body, reducing the volume and excessive pressure in the ventricular system of the brain. Drains located in the atrium of the heart, the pleural cavity and the peritoneal cavity have been described. Currently, the ventriculoperitoneal shunt is preferred due to its relative safety and simplicity (2).

Due to the lack of dedicated drains for veterinary medicine, those designed for newborns are used. This method of treatment has not yet been extensively studied in dogs, but the available literature reports a failure rate of $25 \%$ (9). The most common complications reported for people treated in this way are: infections, insufficient cerebrospinal fluid drainage due to disconnection, bending, squashing, leakage or clogging of the drain and excessive drainage of fluid causing collapse of the cerebral ventricles and the subsequent formation of subdural hematoma $(7,9)$.

Despite the relatively high risk of failure, treatment with ventriculoperitoneal drainage is characterized by a higher and usually more sustained effectiveness than pharmacological treatment (5).

In the described cases, ventricular drainage was effective for $\operatorname{dog} 2$. In the case of $\operatorname{dog} 1$, the cause of the deterioration after the drainage procedure could have been a partial or complete obstruction of the ventriculoperitoneal drain and, consequently, a further increase in intracranial pressure due to fluid accumulation and subsequent mechanical destruction of the partially damaged brain structures.

To sum up, it should be noted that congenital hydrocephalus is a challenging disorder for veterinary surgeons in terms of diagnosis and therapy. The fact that it is not frequently reported may result from the limited access to advanced imaging techniques such as MRI or CT in veterinary medicine. The treatment of dogs with diagnosed hydrocephalus should not be limited to euthanasia. Although difficult and expensive, surgical treatment is very effective and in many cases, as with $\operatorname{dog} 2$, it allows the animal to function normally for many years.

\section{References}

1. Brown J. A., Rachlin J., Rubin J. M., Wollmann R.: Ultrasound evaluation of experimental hydrocephalus in dogs. Surg. Neurol. 1984, 22, 273-276.

2. Filgueiras da Rocha R., de Souza Martins C., de Almeida R. M., Silva R. M., de Souza Teixeira da Rocha M., de Paula Firmino F., Maguilnik S., Medeiros L. $Q$.: Long-term evaluation of a new ventriculoperitoneal shunt valve system in a dog. JVECC 2009, 19, 623-628.

3. Harrington M. L., Bagley R. S., Moore M. P.: Hydrocephalus. Vet. Clin. North. Am. Small. Anim. Pract. 1996, 26, 843-856.

4. Hecht $S$., Adams W. H.: MRI of brain disease in veterinary patients. Part 1: Basic principles and congenital brain disorders. Vet. Clin. North. Am. Small. Anim. Pract. 2010, 40, 21-38.

5. Kolecka M., Ondreka N., Moritz A., Kramer M., Schmidt M. J.: Effect of acetazolamide and subsequent ventriculo-peritoneal shunting on clinical signs and ventricular volumes in dogs with internal hydrocephalus. Acta. Vet. Scand. 2010, 57, 49.

6.Laubner S., Ondreka N., Failing K., Kramer M., Schmidt M. J.: Magnetic resonance imaging signs of high intraventricular pressure - comparison of findings in dogs with clinically relevant internal hydrocephalus and asyptomatic dogs with ventriculomegaly. BMC Vet. Res. 2015, 11, 181.

7. Platt S. R., McConnell J. F., Matiasek L.: Imaging diagnosis - ventriculoperitoneal shunt associated infection in a dog. Vet. Radiol. Ultrasound. 2012, $53,80-83$

8. Przyborowska P., Adamiak Z., Jaskolska M., Zhalniarovich Y.: Hydrocephalus in dogs: a review. Veterinarni Medicina 2013, 58 73-80.

9. Shihab N., Davies E., Kenny P. J., Loderstedt S., Volk H. A.: Treatment of hydrocephalus with ventriculoperitoneal shunting in twelve dogs. Vet. Surg. 2011, 40, 477-484.

10. Stefani A. de, Risio L. de, Platt S. R., Matiasek L., Lujan-Feliu-Pascual A., Garosi L. S.: Surgical technique, postoperative complications and outcome in 14 dogs treated for hydrocephalus with ventriculoperitoneal shunting. Vet. Surg. 2011, 40, 183-191.

11. Summers B. A., Cummings J. F., Lahunta A. de: Inflammatory diseases of the central nervous system, [in:] Veterinary Neuropathology. St. Louis, Mosby, MO 1995, 95-188.

12. Thomas W. B.: Hydrocephalus in dogs and cats. Vet. Clin. North. Am. Small Anim. Pract. 2010, 40, 143-159.

13. Thomas W. B.: Nonneoplastic disorders of the brain. Clin. Tech. Small Anim. Pract. 1999, 14, 125-147.

14. Woo D. C., Choi C. B., Nam J. W., Ryu K. N., Jahng G. H., Lee S. H., Lee D. W., Kim S. Y., Kim H. Y., Ahn K. J., Choe B. Y.: Quantitative analysis of hydrocephalic ventricular alterations in Yorkshire terriers using magnetic resonance imaging. Veterinarni Medicina 2010, 55, 125-132.

Corresponding author: prof. dr hab. Lukasz Adaszek, 30 Głęboka St, 20-612 Lublin, Poland; e-mail: ukaszek0@wp.pl 